\title{
The impact of new-onset diabetes after transplantation on survival and major cardiovascular events in Korean kidney transplantation recipients
}

\author{
Jangwook Lee ${ }^{1}$, Dong Hyun Kang ${ }^{2}$, Sehoon Park ${ }^{3}$, Ji Eun Kim ${ }^{4}$ Eunjeong Kang ${ }^{5}$, Yaerim Kim ${ }^{6}$, Sua Lee ${ }^{1}$, Yong Chul Kim ${ }^{1}$, Yon \\ Su Kim${ }^{1}$,Yaeji Lim², Hajeong Lee ${ }^{1}$
}

\footnotetext{
${ }^{1}$ Department of Internal Medicine, Seoul National University Hospital, Seoul, Korea

${ }^{2}$ Department of Applied Statistics, Chung-Ang University, Seoul, Korea

${ }^{3}$ Department of Internal Medicine, Armed Forces Capital Hospital, Seongnam, Korea

${ }^{4}$ Department of Internal Medicine, Korea University Guro Hospital, Seoul, Korea

${ }^{5}$ Department of Internal Medicine, Ewha Womans University Seoul Hospital, Seoul, Korea

${ }^{6}$ Department of Internal Medicine, Keimyung University Dongsan Medical Center, Daegu, Korea
}

Background: New-onset diabetes after transplantation (NODAT) is a frequent complication in kidney transplant (KT) recipients with unfavorable outcomes, although a nationwide study on epidemiology and clinical outcome of NODAT in Korean KT recipients remain rare.

Methods: We identified KT recipients by using the Health Insurance Review and Assessment Service of South Korea from the year of 2008 to 2017. We excluded patients with preexisting diabetes, multi-organ transplantation, and being progressed to graft failure less than 1 year after KT. NODAT was defined as consecutive 30 days prescription history of antidiabetic medication after KT. We analyzed the impact of NODAT on death censored graft failure (DCGF), death without graft failure (DWGF), and major adverse cardiovascular events (MACE) by time-dependent Cox analysis.

Results: Among a total of 16,719 KT recipients, 10,311 were included after exclusion. The $19.8 \%$ of KT recipients were diagnosed to NODAT. The proportion of patients developing NODAT tended to increase, and $64 \%$ of NODAT was diagnosed within the first 6-months after KT. NODAT patients were older, more men, having longer pre-KT dialysis vintages, and being exposed more basiliximab induction and more rejection episodes requiring high-dose steroids treatment after KT. During follow-up, 520 DCGF, 180 DWGF, and 213 MACE events were occurred. NODAT patients showed higher risks of DCGF (adjusted hazard ratio [aHR], 1.87; 95\% confidence interval [Cl], 1.52-2.3; $\mathrm{P}<0.001)$, DWGF (aHR, 1.77; 95\% Cl, 1.28-2.43; $\mathrm{P}<0.001)$, and MACE (aHR, 1.46; $95 \% \mathrm{Cl}$, 1.08-1.96; $P=0.013$ ) than patients without NODAT. Twenty-one percent of NODAT patients could be stopped their anti-diabetic medications after the diagnosis, although this did not affect the clinical outcomes.

Conclusions: About $20 \%$ of diabetes-naive KT recipients were diagnosed with NODAT with a recently increasing pattern. NODAT in $\mathrm{KT}$ recipients affected worse graft and patients outcomes as well as MACE.

Corresponding author: Jangwook Lee

E-mail: dive2inf@gmail.com

(C) The Korean Society for Transplantation

This is an Open Access article distributed under the terms of the Creative Commons Attribution Non-Commercial License (http://creativecommons.org/licenses/by-nc/4.0/) which permits unrestricted non-commercial use, distribution, and reproduction in any medium, provided the original work is properly cited. 